\title{
Comparison of neural activity that leads to true memories, false memories, and forgetting: An fMRI study of the misinformation effect
}

\author{
Carol L. Baym and Brian D. Gonsalves \\ University of Illinois at Urbana-Champaign, Urbana, Illinois
}

\begin{abstract}
False memories can occur when people are exposed to misinformation about a past event. Of interest here are the neural mechanisms of this type of memory failure. In the present study, participants viewed photographic vignettes of common activities during an original event phase (OEP), while we monitored their brain activity using fMRI. Later, in a misinformation phase, participants viewed sentences describing the studied photographs, some of which contained information conflicting with that depicted in the photographs. One day later, participants returned for a surprise item memory recognition test for the content of the photographs. Results showed reliable creation of false memories, in that participants reported information that had been presented in the verbal misinformation but not in the photographs. Several regions were more active during the OEP for later accurate memory than for forgetting, but they were also more active for later false memories, indicating that false memories in this paradigm are not simply caused by failure to encode the original event. There was greater activation in the ventral visual stream for subsequent true memories than for subsequent false memories, however, suggesting that differences in encoding may contribute to later susceptibility to misinformation.
\end{abstract}

Researchers have long used memory failure as a means to study the capabilities and mechanisms of long-term memory. Indeed, much of our understanding of memory comes from comparing successful memory formation, storage, and retrieval with memory failures of varying degrees, from everyday forgetting to dense amnesia (Cohen \& Eichenbaum, 1993; Eichenbaum \& Cohen, 2001; Johnson \& Raye, 1981; Schacter, 1996, 2001; Wixted, 2004). Failures of memory, however, are not restricted to normal or pathological forgetting, in that memory failures may involve misremembering events (i.e., source confusions or false memories).

Such false memories have been extensively studied behaviorally (for reviews of behavioral results, see Loftus, 2005; Mitchell \& Johnson, 2000; for reviews of neuroimaging results, see Mitchell \& Johnson, 2009; Schacter \& Slotnick, 2004). One way false memories may arise is when events that occur between initial encoding and later retrieval distort memory for the original event. For example, Loftus, Miller, and Burns (1978) presented participants with photographs (depicting an original event) and later introduced verbal information that was inconsistent (misinformation) with what was depicted in some of those photographs. Results from subsequent queries about the content of the photographs showed that misinformation could lead to misremembering of the original event.

Okado and Stark (2005) were, to our knowledge, the only group to investigate the misinformation effect with functional neuroimaging. They used a modification of the misinformation procedure in combination with a subsequent memory analysis, in which brain activity during encoding was sorted on the basis of later memory outcome. In an original event phase (OEP), participants were shown photographs depicting an original event, and in a misinformation phase (MP), they were shown photographs of what was ostensibly the same event but in fact included some modified photographs. Behaviorally, participants showed reliable false memory formation, often mistakenly reporting the source of the misinformation as the original event. Region-of-interest (ROI) analyses showed two main patterns within the medial temporal lobes (MTLs): One set of regions, the left hippocampus tail and left perirhinal cortex, showed more activity during the phase of encoding that would subsequently be remembered (either the OEP or the MP). Another set of regions, including the right hippocampus head and body, left hippocampus body, and left parahippocampal cortex, showed less activity during the phase that would subsequently be remembered (Okado \& Stark, 2005). Importantly, these results suggested that the brain networks that are engaged during encoding of an event play a role in determining the later memory outcome-either accurate remembering of the original source of an event or a source misattribution, in this case when information viewed in the second set of slides was misattributed to the original event. 
The results of Okado and Stark (2005) provided some evidence that the engagement of brain systems important for successful memory encoding provides protection against the effects of misinformation, in that MTL activation associated with later true memory outcomes may have reflected creation of a more robust and enduring memory trace. The present experiment further explored subsequent memory effects related to brain activity during an OEP and an MP, using verbal misinformation, as is more common in behavioral studies of the misinformation effect (Loftus, 2005). Using verbal misinformation also allowed us to examine the potential role of visual imagery during the MP in leading to later false memories in the misinformation paradigm (Dobson \& Markham, 1993).

The present study addressed two central questions. First, can brain activity during encoding of the original event predict susceptibility to or resistance to later misinformation? From the data reported by Okado and Stark (2005), one would predict that false memories arise when the original event is encoded poorly or not at all, leading people to later readily accept misinformation and use it to fill in gaps in memory. Thus, the OEP in trials that lead to later accurate remembering should be associated with relatively higher levels of activation in regions associated with successful encoding - such as those in the MTLthan the OEP in trials leading to later false memories, as was found by Okado and Stark (2005). A second possibility is that misinformation is more likely to take hold in instances when the person has some memory for the original event that perhaps lacks sufficient detail. Under this account, the OEP in trials leading to later false memories may be associated with levels of activation in some brain areas (but not others) that are similar to the levels of activation in the OEP of trials leading to later accurate memories. Alternatively, they may show an intermediate level of activation that falls between that in trials leading to accurate memories and that in trials leading to forgetting. One goal of the present study was to investigate the levels of encoding activation during the OEP as a function of later memory outcome in order to distinguish between these two possibilities.

A second important question we aimed to address concerns which neural and psychological events during presentation of misinformation lead to later reality-monitoring errors and consequently false memories (Johnson \& Raye, 1981). Consistent with the reality-monitoring framework described by Johnson and Raye, there is considerable behavioral evidence that imagery can contribute to false memories (see, e.g., Garry, Manning, Loftus, \& Sherman, 1996; Goff \& Roediger, 1998; Hyman \& Billings, 1998; Johnson, Foley, \& Leach, 1988), including in the misinformation paradigm (Dobson \& Markham, 1993). Gonsalves et al. (2004) were among the first to report using fMRI to examine the neural activity associated with the formation of false memories related to reality-monitoring errors (see also Gonsalves \& Paller, 2000). Results showed that the formation of false memories was associated with greater activity in anterior cingulate cortex, precuneus, and right inferior parietal cortex than were correct rejections. Other studies of visual imagery have found activa- tion in these same regions (Ishai, Ungerleider, \& Haxby, 2000; Kensinger \& Schacter, 2005; Kosslyn \& Thompson, 1999; Suchan et al., 2002). Taken together, these results suggest that engagement of brain regions involved in visual imagery can lead to an increased rate of false memory formation through errors in reality monitoring. If this is the case, then one might expect to see greater activation in these regions during the MP for items associated with later false memories.

In short, we adapted the subsequent memory misinformation paradigm of Okado and Stark (2005) to examine how the presence of verbal misinformation and visual imagery may affect formation of false memories. On the basis of Okado and Stark's (2005) design, the paradigm presented here included an OEP in which participants viewed photographs; rather than presenting another set of photographs during the MP, as in Okado and Stark (2005), the MP in the present experiment consisted of written descriptions of the original photographs, some of which contained misinformation.

\section{METHOD}

\section{Participants}

Participants were recruited through local advertisements in the University of Illinois community. Participants were screened for contraindications to MRI examination. Additionally, individuals with a history of serious cardiovascular or neurological disorders were excluded from the study. After an explanation of the study was given, informed written consent was obtained from all participants prior to initiation of the study. This study was approved by the Institutional Review Board of the University of Illinois at UrbanaChampaign.

The study included 18 right-handed, native English-speaking adults (9 male, 9 female), $19-27$ years of age $(M=22.61)$. Apart from the 18 participants included in the study, 2 additional participants were excluded from analysis due to failure to complete the MP or the memory tests. One participant failed to complete study for the final vignette, so trials from that vignette were not included in analysis for that participant. All participants were financially compensated for their involvement in the study.

\section{Stimuli}

Stimuli for the OEP were 12 vignettes of 50 photographs each, portraying actors engaging in routine daily activities such as renting a movie or preparing dinner. Photographs were sized to $800 \times$ 600 pixels each and were presented on a black background. Presentation of each vignette was preceded by a title slide showing a photograph of the main character(s) of that vignette, along with a short description of what the vignette would depict (Figure 1). Stimuli for the MP were written descriptions of each photograph varying between $4-11$ words each $(M=6.23)$. The written descriptions were presented in white lowercase lettering on a black background. Stimuli were divided into three trial types: critical, control, and generic items. Critical items were stimuli in which a detail from the photograph in the OEP was altered in the written description presented in the MP (Figure 1). Control items were stimuli in which a matching detail was presented in both the OEP and MP (Figure 1). Importantly, subsequently tested details from critical and control items were seen in only one slide across the entire vignette within each phase to control for exposure effects. Generic items were the photographs in the OEP and the written descriptions in the MP that comprised the remaining stimuli (Figure 1), and, as in Okado and Stark's (2005) paradigm, they were included primarily to create coherent stories and were not tested. Within each vignette, there were 12 critical trials, 6 control trials, and 32 generic items, for a 


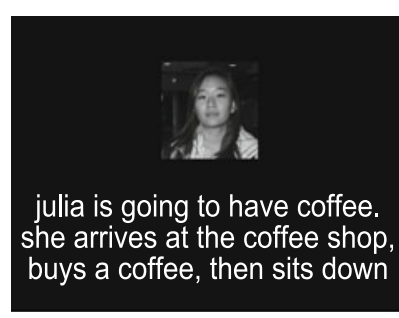

$10 \mathrm{sec}$

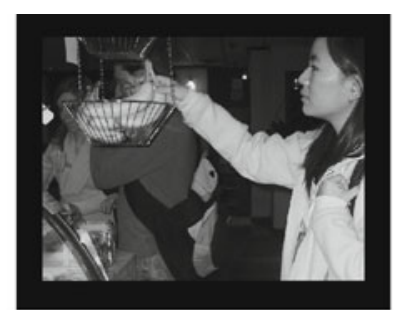

$2,250 \mathrm{msec}$

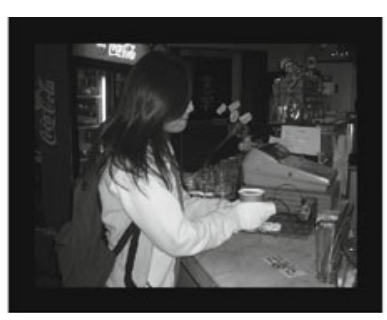

$2,250 \mathrm{msec}$

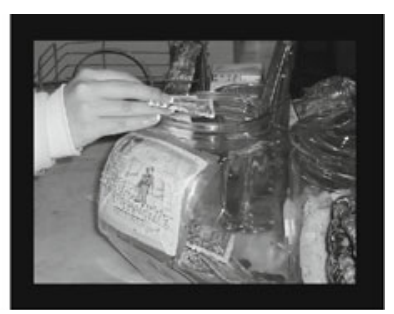

$2,250 \mathrm{msec}$

B

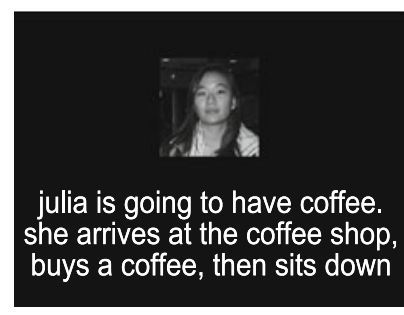

Title

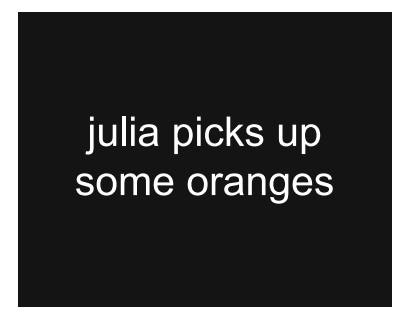

Critical

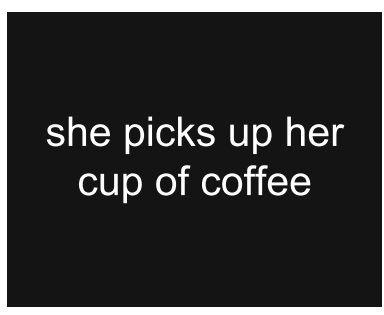

Generic

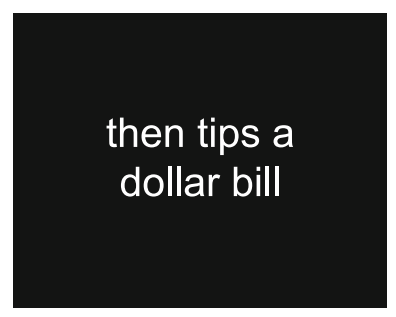

Control

Figure 1. Task design. (A) Trial progression for the original event phase, including a critical item, a generic item, and a control item. (B) Trial progression for the misinformation phase. Written descriptions are enlarged for illustration purposes.

total of 144 critical trials, 72 control trials, and 384 generic items in the experiment overall. Only critical and control items were tested during subsequent item and conflict memory tests. Specific critical and control items were counterbalanced across three groups of participants, so that each item was presented as a critical item in two of the three groups. Results showed no differences among counterbalances in proportion of false memories formed $[F(2,10)=1.003$, $p=.41]$. A repeated measures ANOVA showed that word counts in MP stimuli were matched within vignette across the three trial types (critical, control, generic) $[0.46<F(2,10)<2.39$, all $p \mathrm{~s}>.10]$.

\section{Procedure}

The experiment involved two separate study phases: the OEP and the MP, both conducted in the scanner. To familiarize them with the task, participants were given a short practice session in which they were shown 15 photographs prior to the OEP. Before the task began, participants were told they would be viewing 12 sets of photographs that each comprised a story; additionally, they were told there would be a second task in the scanner but were not informed as to what it was. Participants were not told what the experiment was testing, and they were not apprised of the inclusion of misinformation until debriefing at the end of the experiment. To ensure that participants were paying attention to each photographic stimulus during the OEP, they were given a thematic orienting task in which they were asked to look carefully at all stimuli and covertly select the photograph that they believed to be the least fitting for the overall theme of that vignette. Each stimulus was presented for $2,250 \mathrm{msec}$, followed by a fixation cross presented for $250 \mathrm{msec}$ (Figure 1). Stimuli within vignettes were presented in sequential order and interspersed with variable intertrial intervals. Jittered fixation lasted 2,500-7,500 msec and was interleaved throughout the functional blocks in an order determined by OptSeq2, a program designed to optimize power in deconvolution of trials of each condition and fixation (Dale, 1999). Vignettes were presented in three blocks of four, and the order of blocks was counterbalanced across participants. After each block of four vignettes, participants were asked to tell the experimenter via intercom the item that they selected from one of the vignettes in that block as preselected by the experimenter; vignettes were preselected on the basis of a behavioral pilot in which participants were asked to perform the same thematic orienting task. We used these pilot data to guide selection of which vignette to ask fMRI participants about in each block by selecting the vignette for which the fewest pilot participants chose a critical or control item in this task.

Following completion of the OEP, high-resolution T1-weighted MPRAGE structural images were obtained.

After acquisition of anatomical scans, and approximately $45 \mathrm{~min}$ after viewing the first OEP vignette, participants were informed that they would see the same vignettes again with written descriptions instead of photographs (i.e., the MP). Importantly, participants were not informed of the presence of the written descriptions until after completion of the OEP and the anatomical scans. Immediately prior to the start of the MP, participants were given another short practice session that included written descriptions of the same 15 photographs presented in the first practice session. In the MP, participants were instructed to create a mental image of the photograph corresponding to the written description, or, failing that, to imagine what it might look like. Distribution of critical, control, and generic items in the MP was identical to that in the OEP, with each written description corresponding to a specific photograph from the OEP. Additionally, each vignette in the MP included 5 randomly distributed catch trials (for a total of 60 across the entire MP) in which participants were asked to rate their imagery on the previous item on a 4-point scale $(1=$ no imagery; $4=$ perfect imagery $)$. Post hoc analysis showed that ratings were distributed across trial types in approximate proportion to the frequency of each trial type in the experiment as a whole. Vignettes were presented in the same order during the OEP and MP.

After a 19- to $26-\mathrm{h}$ delay $(M=21.67)$, participants returned for memory testing. The test phase consisted of two separate paper-andpencil tests: an item memory recognition test and a conflict test. Participants were first given a surprise item memory test consisting of 216 questions, including all 12 critical items and 6 control items for each vignette. Questions about each vignette were grouped together and were preceded by a reprint of the title slide participants had seen 
during both the OEP and MP. Each question had three response options in random order. For critical items, response options included (1) information that had been shown in the photograph in the OEP (true memory), (2) information that had been shown in the written description in the MP (false memory), and (3) a novel foil (no memory or guessing) (e.g., What kind of fruit did Julia pick up before getting her coffee? (1) bananas, (2) oranges, (3) apples; see Figure 1). For control items, response options included (1) what had been shown in both the photograph in the OEP and the written description in the MP (true memory), (2) a novel foil (foil 1; no memory or guessing), and (3) a separate novel foil (foil 2; no memory or guessing) (e.g., How much did Julia tip the barista? (1) A dollar bill, (2) A few quarters, (3) She did not tip her; see Figure 1). After completing the item memory recognition test, participants were given a surprise conflict test. The conflict test contained 216 questions corresponding to the 216 questions from the item memory recognition test, and it prompted participants to indicate yes or no as to whether they remembered noticing any conflict between the photograph and written descriptions during the study phases for each critical and control item on the item memory recognition test. During this test, participants were able to view their answers from the item memory recognition test but were unable to change them. Both tests were self-paced and untimed, although participants were instructed to answer each question within $10 \mathrm{sec}$. Following completion of both tests, participants were given the Vividness of Visual Imagery Questionnaire (Marks, 1973), Dissociative Experiences Scale (Bernstein \& Putnam, 1986), and Anomalous Experiences Inventory (Kumar, Pekala, \& Gallagher, 1994). These measures were not significantly correlated with behavioral false memory formation or brain activity when used as covariates in fMRI analyses and are therefore not discussed further.

\section{fMRI Data Acquisition}

Imaging was performed using a 3-Tesla Siemens Magnetom Allegra MRI scanner (Siemens Medical Solutions, Erlangen, Germany) at the University of Illinois Biomedical Imaging Center. Participants viewed visual stimuli on a back-projection screen using an angled mirror mounted on the head coil.

After acquisition of a T2 localizer scan, three functional gradient echo-planar imaging (EPI) runs were collected, each $12 \mathrm{~min} 30 \mathrm{sec}$ long (repetition time $=2,500 \mathrm{msec}, \mathrm{TE}=35 \mathrm{msec}$, 40 interleaved oblique coronal slices, $0.6-\mathrm{mm}$ interslice gap, $3 \times 3 \times 3 \mathrm{~mm}^{3}$ voxels, flip angle $=80^{\circ}$, FOV $=190 \mathrm{~mm}, 298$ volumes per run). Oblique slice acquisition perpendicular to the main axis of the hippocampus was used to minimize susceptibility artifacts during fMRI data acquisition. Slices were positioned to ensure complete coverage of the occipital lobe, at the expense of excluding the frontal poles for participants for whom whole-brain coverage was not possible. Four volumes from the start of each functional scan were removed from analysis to account for magnetic field equilibration. Following the first set of three functional scans (OEP), high-resolution T1 MPRAGE anatomical images were acquired. The three functional runs following anatomical scanning (MP) consisted of 318 volumes per run for a run length of $13 \mathrm{~min} 20 \mathrm{sec}$ and otherwise used parameters identical to those of the OEP functional runs.

\section{fMRI Data Analysis}

Data were preprocessed using SPM5 (Wellcome Department of Cognitive Neurology, London). For each participant, functional images were adjusted for interleaved slice acquisition and were then subjected to affine motion correction. The resulting images were visually inspected for quality of motion correction. Functional volumes were then normalized to the SPM EPI template and resampled to $3 \times 3 \times 3 \mathrm{~mm}^{3}$ voxels. T2-weighted localizer images were then coregistered to the mean EPI volume across runs, and highresolution T1 MPRAGE images were coregistered to T2-weighted images. Again, images were visually inspected following coregistration for quality assurance. Finally, functional images were smoothed with an 8-mm full-width at half-maximum isotropic Gaussian kernel to reduce noise.
Functional data were modeled using the general linear model in SPM5. Event-related fMRI timeseries data were convolved with the canonical hemodynamic response function, which was time-locked to the onset of each photograph or written description. These functions were then used as covariates in the general linear model, along with time-derivative basis functions for each condition, trend effects, and run effects. Least-square parameter estimates of the peak of the hemodynamic response function for each condition were computed for each participant and were then subjected to individual contrasts. These contrasts were then submitted to one-sample $t$ tests at the group level, treating subject as a random effect. All fMRI analyses used a subsequent memory procedure (Brewer, Zhao, Desmond, Glover, \& Gabrieli, 1998; Wagner et al., 1998) in which volumes collected during the OEP and MP were sorted into conditions on the basis of individual responses on the subsequent paper-andpencil item memory recognition test. Epochs in which participants were viewing title information (OEP and MP) or were providing imagery ratings (MP only) were included in the model as regressors of no interest and were not included in analysis. Generic trials were included only in general contrasts of all task-related activity with fixation (OEP $>$ fixation and MP $>$ fixation). All whole-brain analyses are reported at a threshold of $p<.001$, uncorrected, with a 5-contiguous-voxel extent threshold.

ROI analyses were performed using MarsBar 0.41 for SPM5 (Brett, Anton, Valabregue, \& Poline, 2002) and were based on unbiased contrasts of all task-related activity compared with fixation at a threshold of $p<.001$, uncorrected, with a 5-contiguous-voxel extent threshold. In areas of activation that covered multiple functional regions, functional activations were combined with anatomical masks as defined by the Automated Anatomical Labeling Atlas (Tzourio-Mazoyer et al., 2002). Coordinates listed are center of mass in Montreal Neurological Institute space for anatomically masked functional clusters (Evans et al., 1993). ROIs were then submitted to finite impulse response timeseries analysis for the first eight repetition times $(20 \mathrm{sec})$. Peak BOLD responses were calculated for each condition for each ROI and were then subjected to repeated measures ANOVA $F$ tests to compare activity across conditions. If significant, $F$ tests were followed by planned pairwise comparisons using singledegree-of-freedom $F$ contrasts in SuperAnova (Abacus Concepts).

\section{RESULTS}

\section{Behavioral Results}

Critical trials were divided into three conditions on the basis of memory outcome: subsequent true memories $\left(\right.$ True $\left._{\text {crit }}\right)$, subsequent false memories (False crit $_{\text {f }}$ ), and subsequent forgetting (Foil crit $_{\text {) }}$. Control trials were divided into two conditions: subsequent true memories $\left(\right.$ True $_{\text {ctrl }}$ ) and subsequent forgetting (Foil ${ }_{\text {ctrl }}$ ), which was collapsed across the two foils for statistical analyses. A one-way repeated measures ANOVA of condition with five levels was performed on the subsequent memory recognition data $[F(4,68)=136.63, p<.0001]$. Planned pairwise comparisons between True $_{\text {crit }}$ and False crit $_{\text {che }}$, between True $_{\text {crit }}$ and Foil crit $_{\text {, between False }}$ crit and Foil crit, between True $_{\text {ctrl }}$ and Foil ${ }_{\text {ctrl }}$, and between True ${ }_{\text {crit }}$ and True ${ }_{\text {ctrl }}$ were all significant $[6.74<t(17)<19.51$, all $p$ s $<.0001$, using Bonferroni correction for multiple comparisons; see Figure 2]. Participants reported noticing conflict on $18.3 \%$ of False crit $_{\text {trials }}(M=8.61, S D=1.55)$ and on $39.5 \%$ of True $_{\text {crit }}$ trials $(M=31.11, S D=4.19)$.

\section{fMRI Results: OEP}

Whole-brain contrasts. Initial whole-brain analyses focused on identifying regions for which activity dur- 


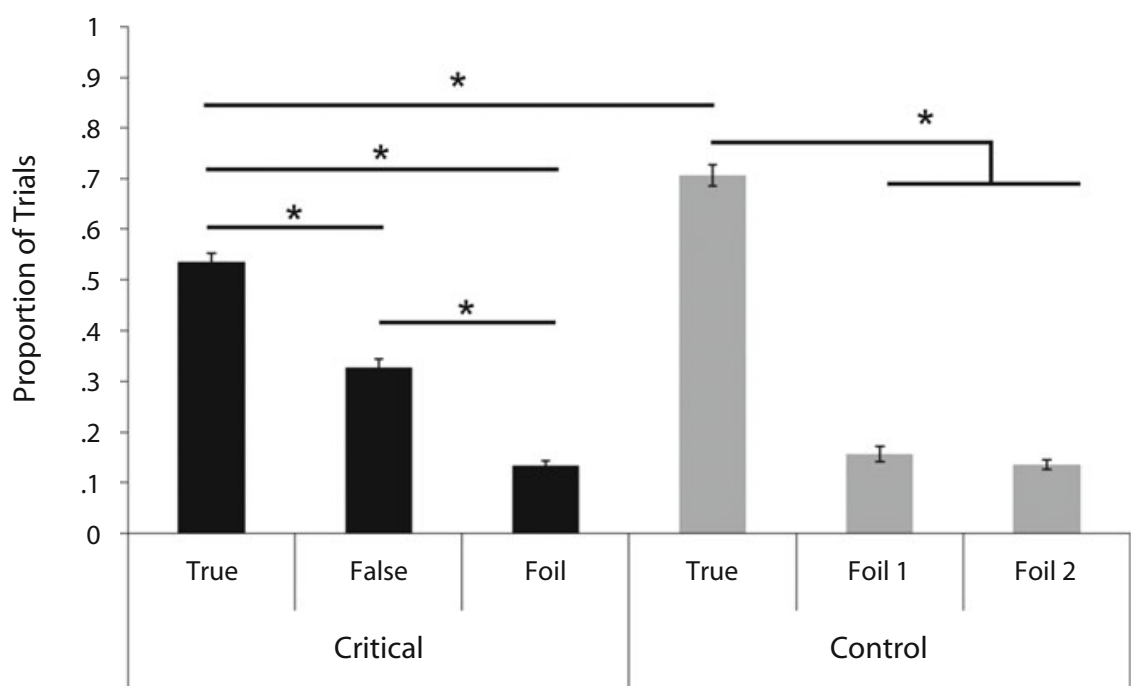

Figure 2. Behavioral responses on the subsequent item memory recognition test broken down by condition within critical (shown in black) and control (shown in gray) categories. Error bars represent within-subjects standard errors. Selections to the foil for control trials are divided into Foil 1 and Foil 2 for illustration purposes.

ing the OEP was associated with subsequent true versus subsequent false memories in the face of misinformation (True crit $_{\text {vs. }}$ False ${ }_{\text {crit }}$ ) in order to assess globally how brain activity during viewing of the OEP might differ for later true and false memories. Clusters of activity in left fusiform gyrus (BA 20/37) and in a region spanning right superior temporal gyrus and lateral occipital cortex (BA 19/39; Figure 3) were greater for subsequent true memories than for subsequent false memories (True $_{\text {crit }}>$ False $_{\text {crit }}$ ). Conversely, a region in left insula (BA 45) was significantly more active for subsequent false memories than for subsequent true memories (False crit $>$ True $_{\text {crit }}$; see Figure 3).

Next, in order to identify regions in which there was greater activity for subsequent false memories than subsequently forgotten items during the OEP, a whole-brain contrast of regions for which activity for False ${ }_{\text {crit }}$ was

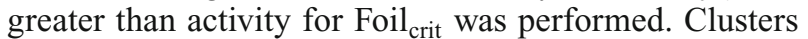
of activity were observed in left inferior frontal gyrus pars operculum (IFG Oper; BA 44), right midcingulate (BA 24), right postcentral gyrus (BA 4), and left superior temporal gyrus (BA 22). Finally, a contrast of activation of subsequent true memories with subsequent forgetting $\left(\right.$ True $_{\text {crit }}>$ Foil $\left._{\text {crit }}\right)$ yielded clusters of activity in right IFG (BA 44), right postcentral gyrus (BA 4), and bilateral fusiform gyrus extending into the posterior temporal cortex (BA 37/39).

ROI analyses. To examine potential differences between subsequent memory type during the OEP, a set of ROIs was defined on the basis of a general contrast of average activation for the OEP compared with fixation $\left(\left[\right.\right.$ True $_{\text {crit }}$, False $_{\text {crit }}$, Foil $_{\text {crit }}$, True $_{\text {ctrl }}$, Foil $_{\text {ctrl }}$, generic $]>$ fixation). Epochs in which participants viewed title screens were modeled as regressors of no interest and were not included in this contrast. From this set of regions generally active while participants viewed scenes, we selected a set of a priori ROIs on the basis of regions implicated in scene encoding in previous subsequent memory studies, including MTL, frontal lobe, and visual cortical regions (Brewer et al., 1998; Gonsalves et al., 2004; Okado \& Stark, 2005; Wagner et al., 1998), which included right hippocampus, bilateral parahippocampus (BA 36/37), right IFG pars triangularis (IFG Tri; BA 45/46), right IFG Oper (BA 44), and right precentral gyrus (BA 9; Figure 4). A region $\times$ condition repeated measures ANOVA across the three frontal regions and subsequent memory accuracy for critical trials showed significant main effects of condition $[F(2,34)=$ $4.76, p<.02]$ and region $[F(2,34)=10.76, p<.0001]$ but no significant interaction $[F(2,34)=0.16, p<.96]$. Collapsing across these frontal regions, results showed significant pairwise differences for True crit versus Foil $_{\text {crit }}[F(1)=$ 14.24, $p<.0006]$ and for False crit $_{\text {versus Foil }}$ crit $[F(1)=$ $7.47, p<.01]$. There was no significant difference between True $_{\text {crit }}$ and False crit $_{\text {cit }}[F(1)=1.08, p<.31]$. Similarly, a region $\times$ condition repeated measures ANOVA grouping the three MTL regions and subsequent memory accuracy for critical trials showed significant main effects of condition $[F(2,34)=7.60, p<.002]$ and region $[F(2,34)=3.421$, $p<.04]$ but no significant interaction $[F(2,34)=0.55$, $p<.70]$. Collapsing across MTL regions showed the same pattern of results as that for the frontal cortex, with significant pairwise differences for True crit $_{\text {versus Foil }}[F(1)=$ 9.16, $p<.005]$ and False crit versus Foil $_{\text {crit }}[F(1)=4.16$, $p<.049$ ] and no significant difference between True crit $_{\text {t }}$ and False crit $[F(1)=0.97, p<.33]$.

\section{fMRI Results: MP}

Whole-brain contrasts. Similarly to the whole-brain analysis in the OEP, whole-brain analysis in the MP focused on comparing later true memories with later false memories to assess whether differences during this phase of viewing predicted accurate remembering or misremem- 


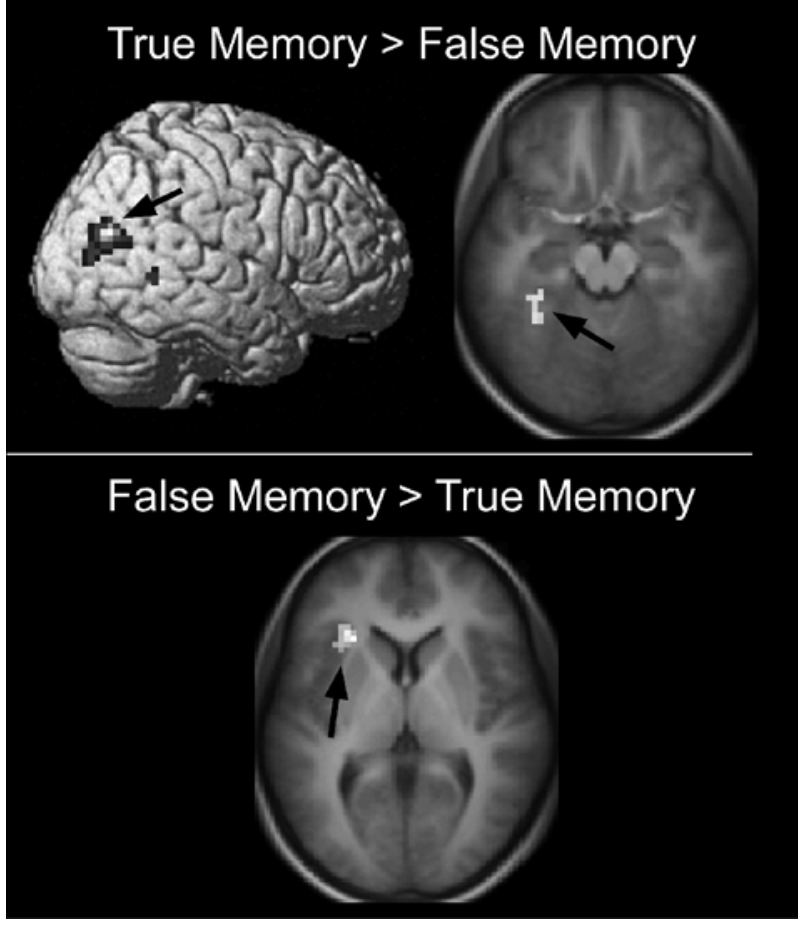

Figure 3. The top panel shows voxel-based activation for subsequent true memories $\left(\right.$ True $_{\text {crit }}$ ) greater than subsequent false memories (False crit $)(p<.001$, five contiguous voxels). Rendering shows a cluster spanning the right superior temporal gyrus and lateral occipital cortex (BA 19/39). Section $(Z=-15)$ shows a cluster in the left fusiform gyrus (BA 20/37). The bottom panel shows voxel-based activation for the reverse contrast-when False $_{\text {crit }}$ was greater than True crit $(p<.001$, five contiguous voxels, $Z=6$ ) - showing a cluster in the left insula (BA 45).

bering of the original photographs. The only significant activation in this contrast was in several regions of the default network along the midline-posterior cingulate cortex (BA 31) extending into left precuneus/cuneus (BA 7) and left anterior cingulate cortex (BA 32) - showed greater activity for subsequent true memories than for subsequent false memories (True $_{\text {crit }}>$ False $_{\text {crit }}$ ). There was no statistically significant activation detected for the reverse contrast, apart from 5 voxels in white matter.

There was an insufficient number of trials in which participants noticed conflict and reported false memories $(M=9.05)$ to be able to analyze differences in activity among false memories with and without awareness of conflict.

ROI analyses. Given that this paradigm reliably created behavioral false memories, we were interested in further exploring how activity in particular brain regions differed across conditions in the MP, particularly for subsequent true and false memories. As a first pass, ROIs were defined in the general contrast of activation for all conditions during this phase with fixation (i.e., activity for True crit, False $_{\text {crit }}$, Foil crit $_{\text {, True }}$ ctrl, and Foil ctrl $_{\text {greater }}$ than that related to fixation). Epochs in which participants viewed title screens or provided imagery ratings were modeled as regressors of no interest and were not included in this contrast. Among critical trials, activity in left IFG Tri (BA 45) followed the predicted pattern of greatest activity for True crit $_{\text {and }}$ False crit $_{\text {compared with }}$ trials in which participants later endorsed the foil (Foil crit $_{\text {) }}$, although differences were not statistically significant $[F(2,34)=1.044, p<.36]$. ROIs were also functionally defined and anatomically masked for regions in left IFG Oper, left IFG pars orbitalis (IFG Orb), left middle temporal cortex, bilateral fusiform gyrus, bilateral hippocampus, and bilateral parahippocampus. Patterns of results across these ROIs were inconsistent and showed no significant differences among conditions.

Previous research has shown visual imagery to be related to an increased false memory rate (Gonsalves et al., 2004; Kensinger \& Schacter, 2005). In the present experiment, it may be the case that more vivid imagery in response to misinformation during the MP led people to form strong visual representations of the scenario described by the misinformation, leading them to later mistake these visually rich memories for memory of what they saw during the OEP. Thus, to examine the effects of imagery on brain activity related to false memory formation in this task, trials for which participants made online judgments of imagery quality during the MP were divided into lowimagery $($ rating $=1$ ) and high-imagery (rating $=4$ ) conditions. One participant was excluded from this analysis due to insufficient trials in the high-imagery condition. A contrast of high-imagery greater than low-imagery trials yielded one six-voxel cluster in right IFG Orb (BA 11) and two small clusters in white matter. No regions were significantly more active for low-imagery trials than for high-imagery trials.

\section{DISCUSSION}

Overall, the version of the misinformation paradigm used here led to reliable behavioral creation of false memories. When details from the OEP and the MP conflicted, participants reported true memories (successfully choosing the version from the OEP) on $53.6 \%$ of trials, false memories (choosing the misinformation version) on $32.9 \%$ of trials, and no memory (choosing a nonpresented foil) on $13.5 \%$ of trials (see Figure 2), generally replicating the behavioral findings of Okado and Stark (2005) using a similar design. Thus, although participants had accurate memory for the original event in the majority of instances, in a significant proportion of cases misleading information did affect their subsequent memory reports of the original event, consistent with previous findings that misinformation can alter or interfere with episodic memories (Loftus, Schooler, \& Wagenaar, 1985; McCloskey \& Zaragoza, 1985).

Our primary interest was how the brain activity that was engaged during encoding of an event - and during exposure to misinformation about that event - was related to true versus false memory outcomes in the later memory test for the original event. In particular, we addressed two main questions: (1) Is there neural evidence that the quality of original encoding offers resistance or susceptibility to later misinformation and, thus, false memories? 
Right Frontal Cortex

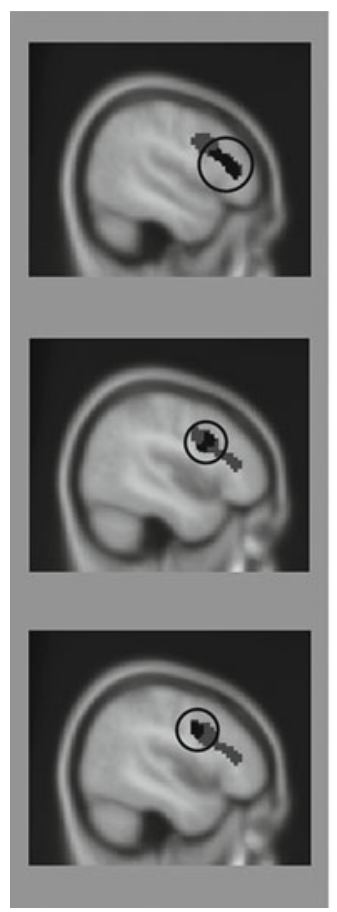

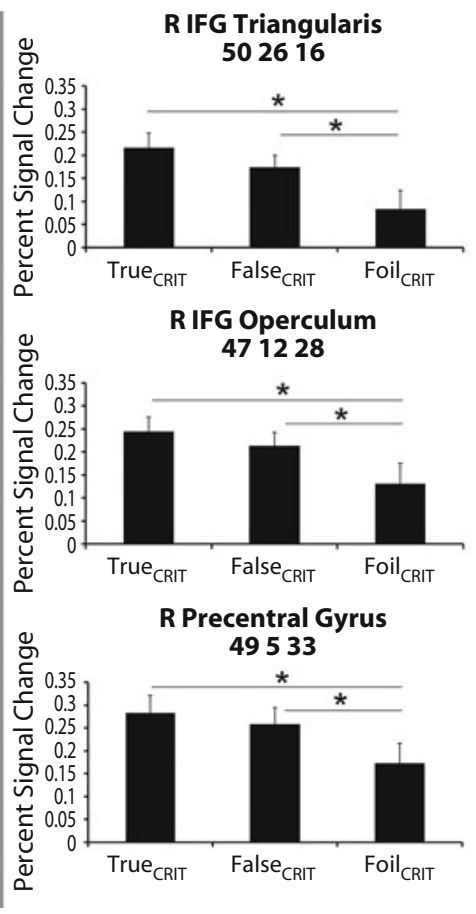

Medial Temporal Lobes

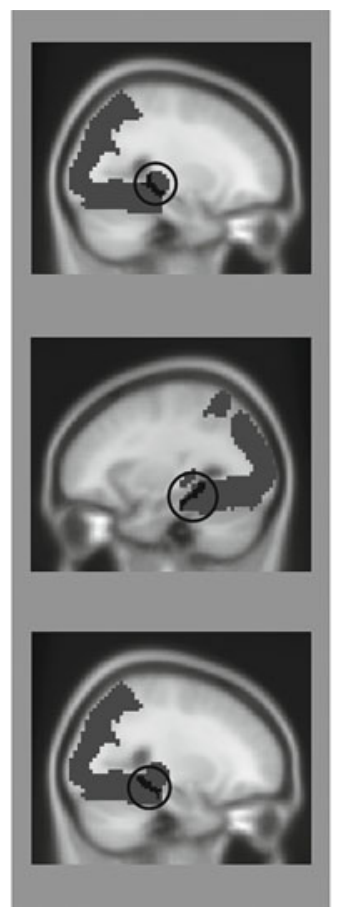

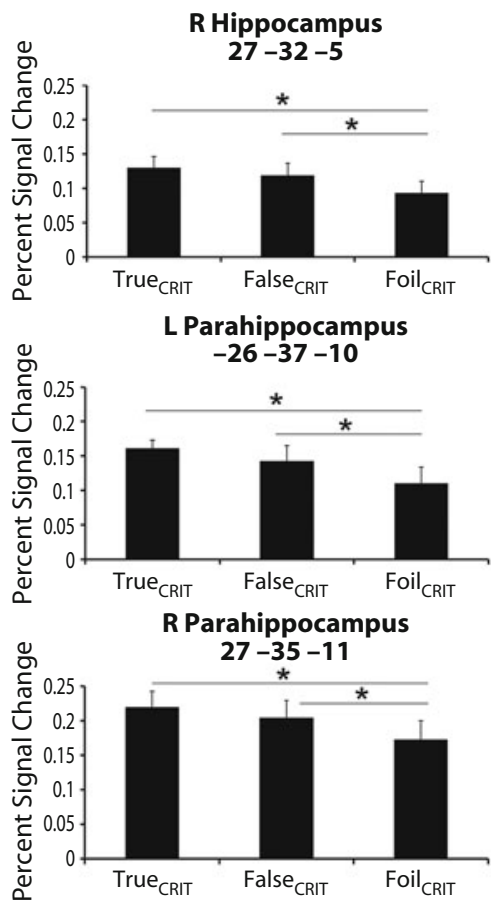

Figure 4. Voxel-based activation in the original event phase for all trials relative to fixation for the right frontal cortex and bilateral medial temporal lobes $(p<.001$, five contiguous voxels). Dark gray indicates a thresholded activation cluster. Black indicates an anatomically masked functional activation. Frontal regions of interest (ROIs): right inferior frontal gyrus (R IFG) triangularis, $X=$ 50; R IFG operculum, $X=45$; right precentral gyrus, $X=46$. Medial temporal lobe ROIs: right hippocampus, $X=27$; left parahippocampus, $X=-30$; right parahippocampus, $X=27$. Coordinates shown indicate the center of mass for the anatomically masked region. Bar graphs depict average activity for the black region for the peak time point of a finite impulse response time course. Error bars represent within-subjects standard errors. True crit $_{\text {, subsequent true memories; False }}$ crit $_{\text {, subsequent false memories; Foil }}$ crit, $_{\text {, }}$ subsequent forgetting.

(2) What neural mechanisms during exposure to verbal misinformation predict subsequent false memories?

With respect to the first question, in the direct contrast of OEP trials that led to later true memories with those that led to false memories of misinformation items, greater activity was observed in occipital and temporal visual processing regions, including fusiform cortex (Figure 3). Fusiform cortex is part of the ventral visual stream, with biases toward processing objects in the fovea (Kastner, De Weerd, Desimone, \& Ungerleider, 1998). In the present experiment, the critical trials in this contrast all had a central object or action of interest that was manipulated in the MP, so that during the item memory recognition test, participants could select the original object, the object described during the MP, or a novel foil as the detail they had seen during the OEP. During the OEP, increased activity in left fusiform gyrus and right temporal/occipital cortex may have reflected increased attention to visual detail, which was associated with later accurate memory for the critical item(s) and thus resistance to the effects of later misinformation. Encoding-activation differences between true and false memories in the present study may therefore have represented a higher quality of encoding or a greater attention to focal details that supported later accurate remembering in the face of misinformation.

These results also support those of previous behavioral investigations of the misinformation effect in which stronger memory for the original event, usually manipulated by repeating presentation of the original event, resulted in greater resistance to misinformation (Marche, 1999; Pezdek \& Roe, 1995; Sutherland \& Hayne, 2001). In addition, behavioral and neuroimaging studies have found that during memory retrieval, true memories tend to be associated with greater ratings and activation of sensory details, as predicted by the reality-monitoring framework (Johnson \& Raye, 1981; see also Slotnick \& Schacter, 2004). That is, even when subjects have failed to discriminate, true memories have been shown to be associated with more behaviorally measured sensory detail (Mather, Henkel, \& Johnson, 1997; Norman \& Schacter, 1997); moreover, neural responses suggest that retrieval of true memories is associated with greater reactivation of sensory-specific cortex (Cabeza, Rao, Wagner, Mayer, \& Schacter, 2001; Okado \& Stark, 2003; Schacter et al., 1996; Schacter \& Slotnick, 2004; Slotnick \& Schacter, 2004). The present finding that ventral visual areas are more active for subsequent true memories than for subse- 
quent false memories adds to previous findings indicating that an important determinant of true versus false remembering may be the amount of item specific detail that was originally encoded.

The results of ROI analyses targeted toward regions previously shown to be involved in encoding of successfully remembered scenes (Brewer et al., 1998; Stern et al., 1996; Wagner et al., 1998) showed that three functional regions in the right frontal cortex were significantly more active for subsequent true memories than for subsequent forgetting: IFG Tri, IFG Oper, and precentral gyrus (see Figure 4). These results are broadly consistent with previous results from subsequent memory studies, which have found increased activity in right IFG to be related to subsequent memory for scenes (e.g., Wagner et al., 1998). Interestingly, in the present study these right frontal regions also showed significantly more activity for subsequent false memories than for subsequent forgetting, and activation levels did not differ significantly between later true and false memories. Bilateral parahippocampal cortex and right hippocampus also showed more activity for subsequent true and false memories than for subsequent forgetting and no difference between activity for true memories and that for false memories (Figure 4). Parahippocampal cortex is thought to be important for representing contextual information in visual scenes (Bar \& Aminoff, 2003; Eichenbaum, Yonelinas, \& Ranganath, 2007; Powell et al., 2005; Ranganath et al., 2004). The hippocampus, however, is thought to be important for the encoding of the arbitrary relations among the elements of a scene, such as spatial relations among the objects in a scene or the relations between objects and their spatial or temporal context (Davachi, Mitchell, \& Wagner, 2003; Eichenbaum \& Cohen, 2001). Taken together with the present results, this suggests that encoding of contextual information was similar for OEPs in trials that led to later true and false memories, and that such contextual information did not tend to engender resistance to the effects of misinformation.

With regard to the MTL results, it is important to note that it would be reasonable to expect that false memories may arise in situations in which little or no information of any kind is originally encoded. In such a case, when presented with misinformation during the MP, participants had no memory from the OEP to counteract the misinformation and thus may have accepted the misinformation version as accurate. Here, however, we observed significantly greater bilateral MTL activity during original encoding of scenes that led to later false memories than during that which led to subsequent forgetting. In fact, trials leading to later false memory were associated with activation in these regions that was comparable to that in trials that led to later true memories. One potential implication of this result is that misinformation is more likely to take hold in cases where some information has been encoded from the original event, rather than in cases where little or nothing was encoded.

To summarize, fusiform cortex showed greater activity for later true memories than for later false memories, whereas right inferior frontal cortex, parahippocampus, and hippocampus all showed levels of activation for later true memories similar to those for false memories, both of which were greater than those for later forgetting. The differences found between true and false memories in ventral visual regions suggest that differential activity during original encoding in these regions may predict whether visual details will be remembered, leading to a true memory, or will be forgotten, leading to a false memory. Activation in medial temporal regions typically associated with the encoding of context was similar for later true memories and later false memories, however, suggesting general contextual information about the viewed scenes was encoded in both cases despite later differential memory outcomes.

Thus, the identity of the regions that did and did not show differential activation for later true memories and later false memories can perhaps provide some hint as to the type of information encoded during the original event that led to different later memory outcomes. One possibility is that attention directed to the focal object(s), perhaps reflecting stronger encoding of the identity or details of these objects or features, provided protection against the effects of misinformation by providing detailed and accurate memory of critical items. Trials in which the general context of the scene was encoded but the details of the focal object were less well encoded, however, were those trials in which participants were particularly susceptible to misleading information about the exact identity of the focal object(s).

Turning to the MP and our second question of interest, we found greater activity for subsequent true memories than for subsequent false memories during this phase primarily in the default network (Buckner, Andrews-Hanna, \& Schacter, 2008; Schacter, Addis, \& Buckner, 2007), including posterior and anterior cingulate cortex and left precuneus/cuneus. Given that deactivation of the default network has been associated with greater engagement in task-related processing, this observation suggests that participants may not have been effectively encoding the verbal misinformation presented during the MP on trials resulting in true memories. Surprisingly, there were no regions of activation in the opposite whole-brain contrast of subsequent false memories greater than subsequent true memories, which is inconsistent with our prediction that MP trials leading to later false memories would be associated with greater activity in regions associated with visual imagery than would MP trials leading to later true memories.

The lack of differences observed in this phase may have been due to the diversity of processes that were likely taking place during the MP for critical trials. For example, a true memory may have resulted from accurate retrieval during the MP of visual details from the OEP and thus may have activated many visual processing regions. A false memory may have involved the reactivation of some information (e.g., contextual detail) from the OEP in response to misinformation. Furthermore, participants were instructed to form a mental image of the photographs corresponding to verbal descriptions during the MP. This may 
have reduced differences in spontaneous mental imagery that might have otherwise predicted false memories. Because of these factors, the null result in the MP data does not provide evidence for or against the role of imagery in the formation of false memories. Previous studies, however, have provided ample behavioral and neuroimaging evidence that imagery does seem to play a causal role in the elicitation of reality-monitoring errors, wherein imagined events are mistaken for actually perceived events (Durso \& Johnson, 1980; Gonsalves \& Paller, 2000; Gonsalves et al., 2004; Hyman \& Pentland, 1996). The present results emphasize a major difficulty in isolating the contributions of imagery to false memories in response to verbal misinformation - namely, the overlap between brain regions involved in retrieving visual memories of events that did occur and those involved in generating imagery in response to a verbal description of an event that did not occur. Further research will be needed to tease apart visual episodic memory and visual imagery processing in response to misinformation that leads to later true versus false memories.

\section{Conclusion}

In the misinformation paradigm used here, differences in neural activity during original encoding of photographs were predictive of subsequent true memory and false memory and forgetting. In visual-processing regions, greater activity predicted subsequent true memories as opposed to subsequent false memories. In right frontal cortex and bilateral MTL, however, activity was greater for both true and false memories than for forgetting. Thus, although a plausible hypothesis is that false memories will show an original encoding profile similar to that of forgetting, the neuroimaging results suggest instead that the formation of false memories requires that at least some information about the original event be encoded in order for misinformation to affect later memory. The results of the present study suggest that strong encoding of the general contextual frame of an event, combined with weaker encoding of the particular details that turn out later to be important - such as the identity or specific features of objects present during the event-are optimal conditions for the formation of false memories.

\section{AUTHOR NOTE}

C.L.B. was supported by the Department of Defense through the National Defense Science and Engineering Graduate Fellowship Program. We also thank Alex Marquis for assistance with stimulus creation and data collection, Brion Woroch for assistance with data analysis, Neal J. Cohen for helpful comments on design and results, and Marcia K. Johnson and two anonymous reviewers for their insightful comments on the manuscript. Correspondence concerning this article should be addressed to C. L. Baym, Department of Psychology, 603 E. Daniel St., Champaign, IL 61820 (e-mail: cbaym@illinois.edu).

\section{REFERENCES}

BAR, M., \& Aminoff, E. (2003). Cortical analysis of visual context. Neuron, 38, 347-358. doi:10.1016/S0896-6273(03)00167-3

Bernstein, E. M., \& Putnam, F. W. (1986). Development, reliability, and validity of a dissociation scale. Journal of Nervous \& Mental Disease, 174, 727-735.

Brett, M., Anton, J. L., Valabregue, R., \& Poline, J. B. (2002,
June). Region of interest analysis using an SPM toolbox. Presented at the 8th International Conference on Functional Mapping of the Human Brain, Sendai, Japan.

Brewer, J. B., Zhao, Z., Desmond, J. E., Glover, G. H., \& Gabrieli, J. D. E. (1998). Making memories: Brain activity that predicts how well visual experience will be remembered. Science, 281, 1185-1187. doi:10.1126/science.281.5380.1185

Buckner, R. L., Andrews-Hanna, J. R., \& Schacter, D. L. (2008). The brain's default network: Anatomy, function, and relevance to disease. In A. Kingstone \& M. B. Miller (Eds.), The year in cognitive neuroscience 2008 (Annals of the New York Academy of Sciences, Vol. 1124, pp. 1-38). doi:10.1196/annals.1440.011

Cabeza, R., Rao, S. M., Wagner, A. D., Mayer, A. R., \& Schacter, D. L. (2001). Can medial temporal lobe regions distinguish true from false? An event-related functional MRI study of veridical and illusory recognition memory. Proceedings of the National Academy of Sciences, 98, 4805-4810.

Cohen, N. J., \& Eichenbaum, H. (1993). Memory, amnesia, and the hippocampal system. Cambridge, MA: MIT Press.

Dale, A. M. (1999). Optimal experimental design for event-related fMRI. Human Brain Mapping, 8, 109-114.

Davachi, L., Mitchell, J. P., \& Wagner, A. D. (2003). Multiple routes to memory: Distinct medial temporal lobe processes build item and source memories. Proceedings of the National Academy of Sciences, 100, 2157-2162. doi:10.1073/pnas.0337195100

Dobson, M., \& Markham, R. (1993). Imagery ability and source monitoring: Implications for eyewitness memory. British Journal of Psychology, 84, 111-118.

Durso, F. T., \& Johnson, M. K. (1980). The effects of orienting tasks on recognition, recall, and modality confusion of pictures and words. Journal of Verbal Learning \& Verbal Behavior, 19, 416-429.

Eichenbaum, H., \& Cohen, N. J. (2001). From conditioning to conscious recollection: Memory systems of the brain. New York: Oxford University Press.

Eichenbaum, H., Yonelinas, A. P., \& Ranganath, C. (2007). The medial temporal lobe and recognition memory. Annual Review of Neuroscience, 30, 123-152. doi:10.1146/annurev .neuro.30.051606.094328

Evans, A. C., Collins, D. L., Mills, S. R., Brown, E. D., Kelly, R. L., \& Peters, T. M. (1993). 3D statistical neuroanatomical models from 305 MRI volumes. In L. A. Klaisner (Ed.), Proceedings of IEEENuclear Science Symposium and Medical Imaging (pp. 1813-1817). Piscataway, NJ: IEEE Service Center.

Garry, M., Manning, C. G., Loftus, E. F., \& Sherman, S. J. (1996). Imagination inflation: Imagining a childhood event inflates confidence that it occurred. Psychonomic Bulletin \& Review, 3, 208-214.

Goff, L. M., \& Roediger, H. L., III (1998). Imagination inflation for action events: Repeated imaginings lead to illusory recollections. Memory \& Cognition, 26, 20-33.

Gonsalves, B. [D.], \& Paller, K. A. (2000). Neural events that underlie remembering something that never happened. Nature Neuroscience, 3, 1316-1321. doi:10.1038/81851

Gonsalves, B. [D.], Reber, P. J., Gitelman, D. R., Parrish, T. B., MeSUlam, M.-M., \& PAller, K. A. (2004). Neural evidence that vivid imagining can lead to false remembering. Psychological Science, 15, 655-660. doi:10.1111/j.0956-7976.2004.00736.x

Hyman, I. E., JR., \& Billings, F. J. (1998). Individual differences and the creation of false childhood memories. Memory, 6, 1-20.

Hyman, I. E., JR., \& Pentland, J. (1996). The role of mental imagery in the creation of false childhood memories. Journal of Memory \& Language, 35, 101-117.

IshaI, A., Ungerleider, L. G., \& HAXBy, J. V. (2000). Distributed neural systems for the generation of visual images. Neuron, 28, 979-990. doi:10.1016/S0896-6273(00)00168-9

Johnson, M. K., Foley, M. A., \& LEACH, K. (1988). The consequences for memory of imagining in another person's voice. Memory \& Cognition, 16, 337-342.

Johnson, M. K., \& RaYe, C. L. (1981). Reality monitoring. Psychology Review, 88, 67-85.

Kastner, S., De Weerd, P., Desimone, R., \& Ungerleider, L. G. (1998). Mechanisms of directed attention in the human extrastriate cortex as revealed by functional MRI. Science, 282, 108-111. doi:10.1126/science.282.5386.108 
Kensinger, E. A., \& Schacter, D. L. (2005). Emotional content and reality-monitoring ability: fMRI evidence for the influences of encoding processes. Neuropsychologia, 43, 1429-1443. doi:10.1016/j .neuropsychologia.2005.01.004

KossLyn, S. M., \& Thompson, W. L. (1999). Shared mechanisms in visual imagery and visual perception: Insights from cognitive neuroscience. In M. S. Gazzaniga (Ed.), The new cognitive neurosciences (2nd ed., pp. 975-985). Cambridge, MA: MIT Press.

Kumar, V. K., Pekala, R. J., \& Gallagher, C. (1994). The Anomalous Experiences Inventory. Unpublished manuscript, West Chester University, Pennsylvania.

LofTus, E. F. (2005). Planting misinformation in the human mind: A 30 -year investigation of the malleability of memory. Learning \& Memory, 12, 361-366. doi:10.1101/lm.94705

Loftus, E. F., Miller, D. G., \& Burns, H. J. (1978). Semantic integration of verbal information into a visual memory. Journal of Experimental Psychology: Human Learning \& Memory, 4, 19-31.

Loftus, E. F., Schooler, J. W., \& Wagenaar, W. A. (1985). The fate of memory: Comment on McCloskey and Zaragoza. Journal of Experimental Psychology: General, 114, 375-380.

Marche, T. A. (1999). Memory strength affects reporting of misinformation. Journal of Experimental Child Psychology, 73, 45-71. doi:10.1006/jecp.1998.2489

MARKs, D. F. (1973). Visual imagery differences in the recall of pictures. British Journal of Psychology, 64, 17-24.

Mather, M., Henkel, L. A., \& Johnson, M. K. (1997). Evaluating characteristics of false memories: Remember/know judgments and memory characteristics questionnaire compared. Memory \& Cognition, 25, 826-837.

McCloskey, M., \& Zaragoza, M. (1985). Misleading postevent information and memory for events: Arguments and evidence against memory impairment hypotheses. Journal of Experimental Psychology: General, 114, 1-16.

Mitchell, K. J., \& Johnson, M. K. (2000). Source monitoring: Attributing mental experiences. In E. Tulving \& F. I. M. Craik (Eds.), The Oxford handbook of memory (pp. 179-195). New York: Oxford University Press.

Mitchell, K. J., \& Johnson, M. K. (2009). Source monitoring 15 years later: What have we learned from fMRI about the neural mechanisms of source memory? Psychological Bulletin, 135, 638-677. doi:10.1037/a0015849

Norman, K. A., \& Schacter, D. L. (1997). False recognition in younger and older adults: Exploring the characteristics of illusory memories. Memory \& Cognition, 25, 838-848.

OKADO, Y., \& STARK, C. [E. L.] (2003). Neural processing associated with true and false memory retrieval. Cognitive, Affective, \& Behavioral Neuroscience, 3, 323-334.

OKado, Y., \& Stark, C. E. L. (2005). Neural activity during encoding predicts false memories created by misinformation. Learning \& Memory, 12, 3-11. doi:10.1101/lm.87605

Pezdek, K., \& Roe, C. (1995). The effect of memory trace strength on suggestibility. Journal of Experimental Child Psychology, 60, 116128. doi:10.1006/jecp.1995.1034
Powell, H. W. R., Koepr, M. J., Symms, M. R., Boulby, P. A., SaleKHAdDAdi, A., Thompson, P. J., ET AL. (2005). Material-specific lateralization of memory encoding in the medial temporal lobe: Blocked versus event-related design. NeuroImage, 27, 231-239. doi:10.1016/j .neuroimage.2005.04.033

Ranganath, C., Yonelinas, A. P., Cohen, M. X., Dy, C. J., Tom, S. M., \& D'Esposito, M. (2004). Dissociable correlates of recollection and familiarity within the medial temporal lobes. Neuropsychologia, 42, 2-13. doi:10.1016/j.neuropsychologia.2003.07.006

SCHACTER, D. L. (1996). Searching for memory: The brain, the mind, and the past. New York: Basic Books.

SCHACTER, D. L. (2001). The seven sins of memory: How the mind forgets and remembers. Boston: Houghton Mifflin.

Schacter, D. L., AdDis, D. R., \& Buckner, R. L. (2007). Remembering the past to imagine the future: The prospective brain. Nature Reviews Neuroscience, 8, 657-661. doi:10.1038/nrn2213

Schacter, D. L., Reiman, E., Curran, T., Yun, L. S., Bandy, D., MCDermott, K. B., \& Roediger, H. L., III (1996). Neuroanatomical correlates of veridical and illusory recognition memory: Evidence from positron emission tomography. Neuron, 17, 267-274.

Schacter, D. L., \& Slotnick, S. D. (2004). The cognitive neuroscience of memory distortion. Neuron, 44, 149-160. doi:10.1016/j .neuron.2004.08.017

Slotnick, S. D., \& Schacter, D. L. (2004). A sensory signature that distinguishes true from false memories. Nature Neuroscience, 7, 664 672. doi: $10.1038 / \mathrm{nn} 1252$

Stern, C. E., Corkin, S., González, R. G., Guimaraes, A. R., BaKer, J. R., JenNings, P. J., ET AL. (1996). The hippocampal formation participates in novel picture encoding: Evidence from functional magnetic resonance imaging. Proceedings of the National Academy of Sciences, 93, 8660-8665.

Suchan, B., Yágüez, L., Wunderlich, G., Canavan, A. G. M., Herzog, H., Tellmann, L., ET AL. (2002). Neural correlates of visuospatial imagery. Behavioural Brain Research, 131, 163-168. doi:10.1016/ S0166-4328(01)00373-4

Sutherland, R., \& HaYne, H. (2001). Age-related changes in the misinformation effect. Journal of Experimental Child Psychology, 79, 388-404.

Tzourio-Mazoyer, N., Landeau, B., Papathanassiou, D., CriVEllo, F., Etard, O., Delcroix, N., ET AL. (2002). Automated anatomical labeling of activations in SPM using a macroscopic anatomical parcellation of the MNI MRI single-subject brain. NeuroImage, 15, 273-289. doi:10.1006/nimg.2001.0978

Wagner, A. D., Poldrack, R. A., Eldridge, L. L., Desmond, J. E., Glover, G. H., \& Gabrieli, J. D. E. (1998). Material-specific lateralization of prefrontal activation during episodic encoding and retrieval. NeuroReport, 9, 3711-3717.

WiXTED, J. T. (2004). The psychology and neuroscience of forgetting. Annual Review of Psychology, 55, 235-269. doi:10.1146/annurev .psych.55.090902.141555

(Manuscript received July 17, 2009; revision accepted for publication May 13, 2010.) 